\title{
Experimental study of hydraulic equipment operation process
}

\author{
Kamoliddin Rustamov", Samandar Komilov, Mavlon Kudaybergenov, Shamshir Shermatov and Shahzod \\ Xudoyqulov \\ Tashkent State Transport University, Tashkent, Uzbekistan
}

\begin{abstract}
Relevance. When the hydraulic fluid is operated for several cycles, the impact on hydraulic performance and longevity is greatly increased. So, there are several ways to fix this problem. To increase the efficiency of the hydraulic system, it is necessary to study the working processes, the hydraulic systems of the quick-change equipment of the machine with a lawn of the $\mathrm{k}_{\text {shp }}$ are made as a whole, and the equipment of the machine is attached to the suspension device. The complexity of these processes lies in the fact that they allow experimental research and verification of results using mathematical models.

Aim. The purpose of the experimental study in this dissertation is to study the effect of forces acting on the hydraulic system when excavating the hydraulic system of a multipurpose machine and substantiate the parameters by assessing power use efficiency.

Methods. Checking the suitability of the working fluid during earthworks, as well as the mathematical model. In this case, we studied the hydraulic system of a single-bucket excavator, the device of quick-change equipment for a universal machine.

Results. The theoretical work performed has been verified and the confirmation of the operation of a multipurpose machine designed with earth and dozer equipment. The installation of equipment based on TTZ-80 made it possible to carry out experimental research with the most energy-intensive workflow, determining energy efficiency indicators for excavation and leveling.

Conclusion. Comprehensive tests made it possible to conclude that the characteristics of the developed design and the characteristics of the engine of the base machine - the TTZ-80.10 - KM-1 tractor, overcome the resistance encountered during technological operations of a multipurpose machine.
\end{abstract}

\section{Introduction}

The development of the republic's economy is inextricably linked with the expansion of the road network, the growth of existing capacities, maintenance, repair and reconstruction of roads, maintenance and repair of airfields. Implementing these tasks based on high-performance road-building machinery and equipment will significantly save material, energy and labor resources.

The Republic of Uzbekistan is among the countries striving to enter the number of developed countries. The Resolution "On the Strategy of Actions for the Further Development of the Republic of Uzbekistan" states that "the implementation of targeted programs for the development and modernization of road transport, engineering, communication and social infrastructure" [1]. These issues will be resolved through the import of modern equipment from abroad, which means that high costs will have to be incurred. Such machines, produced in the USA, Germany, Sweden, Russia, Ukraine, do not fully correspond to the technological process, and their operation in Central Asia is very expensive due to the use of expensive materials, including universal machines and mechanisms in our country. Research aimed at developing the KM-1 machine tool, the choice of the hydraulic system, parameters of the hydraulic drive of drilling equipment [15-20].

\footnotetext{
*Corresponding author: k.j.rustamov82@gmail.com
} 


\section{Materials and Methods}

In theoretical studies, the theory of mechanisms and machines, the science of hydromechanics, as well as the methods of computational mathematics were used. When researching this area, modern equipment was used and statistical and mathematical methods for planning and processing experimental work. At the same time, there are various types of computational work and the Matlab programming language.

\section{Results and Discussion}

In the course of the experiment, the following tasks were set to study the physical processes occurring in the hydraulic device and the influence of resistance forces on the equipment:

- Checking and adjusting the mathematical model of the hydraulic system, as well as the impact of the drilling rig on the workflow for using the working fluid at the required pressure;

- to conduct experimental research in such conditions and obtain research results.

The purpose of the experiments is to test theoretical studies and confirm the power levels of a multipurpose machine with digging and pushing devices [2-14].

\subsection{Type and application of devices and equipment used in the experiment}

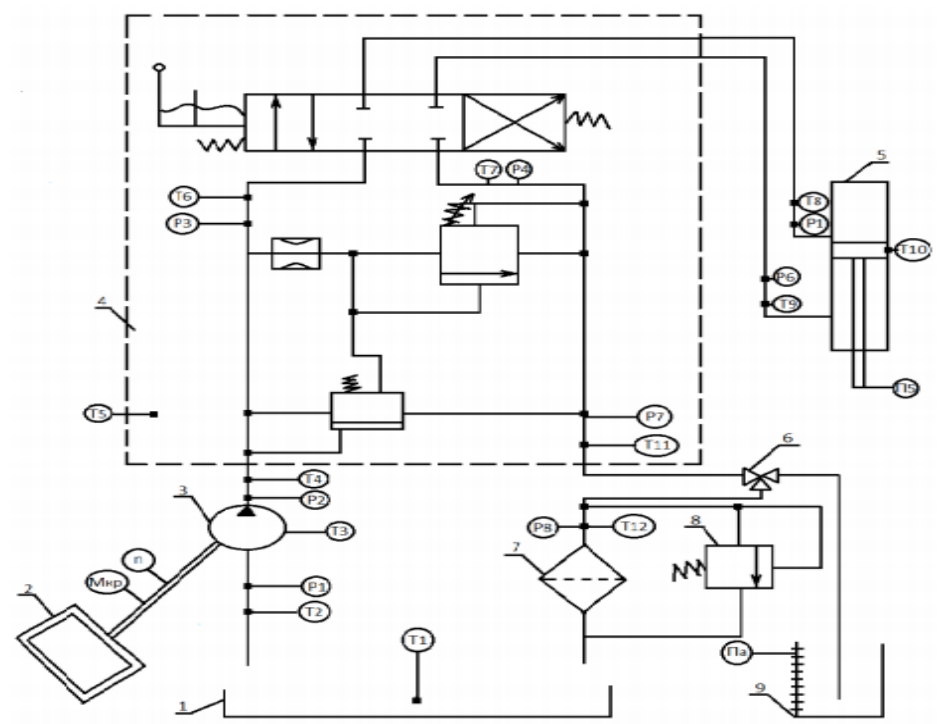

Fig. 1. Approximate (principal) hydraulic scheme of the study

1 is main tank; 2 is measuring motor; 3 is pump NSh-32M4; 4 is distributor R80 3/1 222; 5 is power hydraulic cylinder Ts-55; 6 is three-way valve; 7 is rear filter; 8 is safety valve in the tank; 9 is tank.

$\mathrm{P}$ is pressure sensor; $\mathrm{T}$ is temperature sensor; $\mathrm{P}$ is displacement sensor; $\mathrm{Pa}$ is flow rate sensor; $\mathrm{n}$ is sensor for measuring the number of revolutions; $\mathrm{M}$ is torque sensor

The study of the processes of excavation with a universal machine was carried out to study the operation of the hydraulic system, as well as to determine the regularities of changes in the volumetric and energy properties of systems depending on: a) oil temperature; b) types of oils; c) voltage; d) the number of revolutions of the pump shaft; d) ambient temperature. All recorded parameters (pressure, rpm, temperature, etc.) were recorded from the moment the test bench was launched on an N-115 oscilloscope and other devices.

During the observations, in addition to the above, the following parameters were noted: the time of starting and warming up the engine, the temperature of the oil and coolant, and the time of the forced stop. According to the accepted methodology, the machines are equipped with the following pressure sensors, instruments and equipment: 1. Strain gauge for measuring the torque of the NSh-32M4 hydraulic pump in the drive (Figures 2, $3)$. 


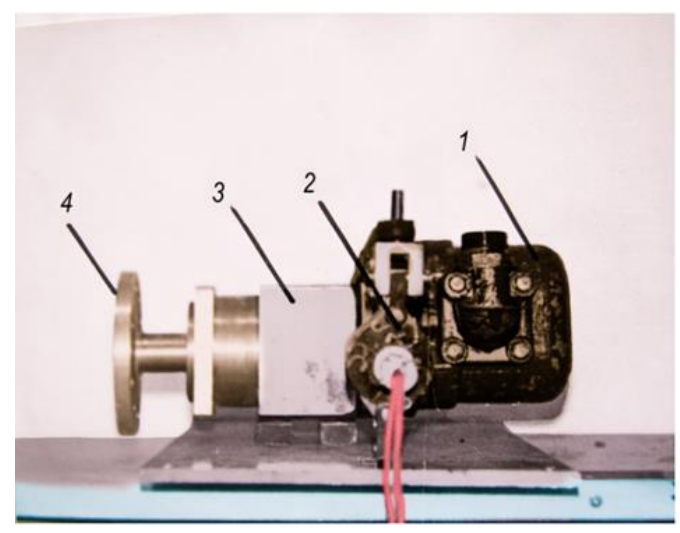

Fig. 2. Strain gauge measuring torque, 1 is pump NSh32M4; 2 is tensometric; 3 is sensor body; 4 is drive flange.

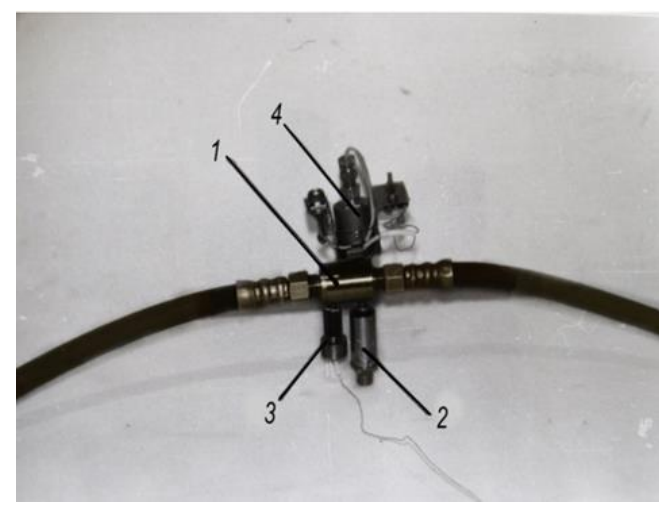

Fig. 3. Installation of pressure and temperature sensors in the pipeline, 1 is conductive bushing; 2 is pressure strain gauge sensor; 3 are temperature sensors; 4 are type ID pressure sensors.

2. Pressure sensor for recording the pressure at the inlet and outlet of the hydraulic pump (fig. 4).
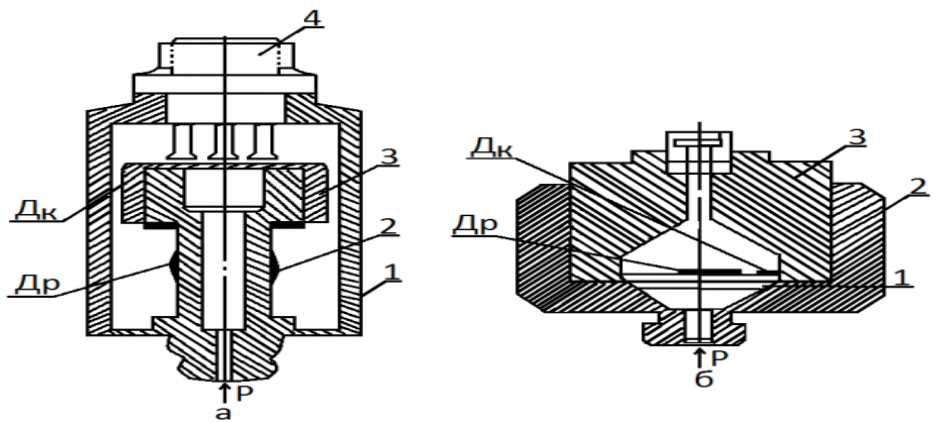

a is tubular: 1 is body; 2 is working pipe; 3 is shutter (plug); 4 is plug connector; $b$ is membrane: 1 is membrane; 2 is sensor body; 3 is concealer; $Д_{\kappa}$ is a sensor compensating the strain gage; $Д_{\mathrm{P}}$ is working strain gauge

Equipment recorded with voltage sensors and power supplies installed in the machine was detected in an experiment using universal machine cables and sensors. Torque and pressure were measured with an ST-23 recorder, which allows recording on six channels. The working fluid temperature was recorded with a PP-63 portable potentiometer.

An oscilloscope operating on N-115 light beams was used as the main recording device; The writing was carried out on a paper cutter UF-67-100, which made it possible to reduce the processing time of materials since the research requires time to work with photography. For measurements using pressure gauges, an $8 \mathrm{AHY} 7 \mathrm{M}$ eight-channel voltage amplifier was used.

The torque value was determined to determine the power consumption of the pump drive. Two types of recording devices were used in the studies - a VISXOM device, a Russian-made torque sensor and a selfrecording small-sized continuously-adjustable device N348, designed to measure and register active and passive movements. The torque sensor is shown in Figure 2. The number of revolutions of the pump shaft was recorded by an induction sensor.

The technical condition of the hydraulic systems was checked by the KI device. The MP-160 type pistonload pressure gauge with a pressure gauge calibration device was used to calibrate the pressure.

Following the requirements of regulatory documents, the determination of the energy value and power consumption of earthmoving machinery is carried out based on measuring their torque and rotation frequency. The operation of all excavator equipment of the experimental machine is provided by 2 gear pumps, and to calculate the energy, it is necessary to determine the power spent on their drive. The design of the drive does not allow for directly measuring the pump torque and then calculating the power consumed by their drive. The current GOST 300067-93 "Excavator single-bucket universal full-revolving" [6, 7, 10] allows you to determine the indicators calculated by indirect measurement methods, based on the characteristics of the tested equipment, in cases where measurement is difficult directly. 


\subsection{Define indicators and process the results}

The power of the gear pump in the drive is determined by the following formula

$$
N=\frac{Q P}{61.2 n}
$$

where: $N$ is drive pump power, $\mathrm{kW}$;

$Q$ is volumetric transfer of the pump, 1 / min;

$P$ is working pressure, $\mathrm{MPa}$;

$n$ is overall pump efficiency.

The pump displacement is determined by the following formula:

$$
Q=\frac{n q}{1000}
$$

where: $q$ is pump performance in one revolution, $\mathrm{cm}^{3}$;

$n$ is rotation speed of the pump drive shaft, $\mathrm{min}^{-1}$.

Load per revolution - " $q$ " and efficiency are the main parameters of the pump. It is known from the technical characteristics of the pumps that the efficiency of both the NSh-32M4 and NSh-100-3 pumps can be calculated to be the same and equal to 0.85 , and the working volumes in the first case are $31.5 \mathrm{~cm}^{3}$ and $32 \mathrm{~cm}^{3}$ in the second, respectively.

Thus, only direct measurement of the operating pressure and rotation speed of the drive shaft is sufficient to determine the power consumed by hydraulic pumps of drilling rigs [11].

The energy assessment was carried out jointly with the Uzbek Research Institute of Agricultural Mechanization and Electrification (UzMEI) based on a special test program approved by the working group of UzMEI and TDTrU following GOST 300067-93 "Single-bucket rotary universal excavator. "[6, 7].

The following parameters were directly measured according to the test program and energy estimation methodology (Table 1).

Table 1. Compliance with test program and energy assessment methodology

\begin{tabular}{|c|l|l|}
\hline № & \multicolumn{1}{|c|}{ Indicators name } & \multicolumn{1}{|c|}{ The size } \\
\hline 1 & Oil pressure & \\
\hline & Hydraulic travel pump outlet pressure & $\mathrm{MPa}$ \\
\hline & pressure at the outlet of the pumping station & $\mathrm{MPa}$ \\
\hline 2 & Rotation frequency & \\
\hline & For engine & $\mathrm{s}-1$ \\
\hline & For pumping station & $\mathrm{s}-1$ \\
\hline 3 & Oil temperature & ${ }^{\circ} \mathrm{C}$ \\
\hline 4 & Time spent on working cycles of earthmoving equipment: & The size \\
\hline
\end{tabular}

Following the established instructions (GOST 300065-93 and GOST 300067-93), tests were carried out using electrical measurement methods of non-electrical quantities and, in particular, electricity meters.

Since the energy was estimated following the average values of the measured parameters, small-sized and recording devices EMA-P were used to measure the measuring voltage (Fig. 9). The equipment was calibrated with a stamp caliber TK-22, and its technical condition was checked during testing [8].

Following the work program, pressure measurements were carried out using TDD-200 voltmeters manufactured by VISXOM (Russia). The sensors were calibrated in an MP-600 calibration device using a standard manometer $250 \mathrm{~kg} / \mathrm{cm} 2$ [9].

The results of the initial and final calibration of the pressure sensors showed that the measurement channels were reduced, and the total errors are within acceptable limits $(+2.5 \%)$. Using an adapter, the sensor was mounted on the NSh-100-3 pumping station.

Analysis of the kinematic diagram of drilling rigs shows that to calculate the rotational speed of the drive shafts of all hydraulic pumps, it is sufficient to measure the rotational speed of the engine crankshaft, and from a technical point of view, this measurement of the rotational speed is the simplest and least expensive.

In this case, the number of gears of the drive of the pumping station $\mathrm{NSh}_{2} 2 \mathrm{M}^{-4}$ is equal. The rated speed of the engine crankshaft is $2100-2200 \mathrm{~min}^{-1}$, which provides the following rotational speeds: pump station NSh-32$\mathrm{M}^{-4}-32.3 \mathrm{~s}^{-1}\left(1940 \mathrm{~min}^{-1}\right)$ and pump NSh-100-3. ... $-22.5 \mathrm{~s}^{-1}\left(1348 \mathrm{~min}^{-1}\right)$. 
To measure the rotational speed of the engine crankshaft, a magnetically controlled contact was used - a reed switch. For this, two magnets 2 were installed on the pulley of the cooling system and the power supply unit mounted on the engine crankshaft, and a rod was installed on the left and right traction beam grilles, including 4 connectors ShR20 P4, equipped with a tongue switch. The Gercon switch is connected to a digital input of the EMA-P device. When the tool rotates the crankshaft, the magnets passing under the button of the shoulder cause the closure of its contacts, the number of which is calculated by a discrete channel [5].

The temperature of the oil in the reservoir was measured by a digital electrothermometer FLUKE-52 (USA), and during the experiments, it was about $52-56^{\circ} \mathrm{C}$. The measuring device was installed on the table, and the length of the connecting wires allowed performing technological operations in the required volumes. EMA-P equipment was powered from the power source of the TTZ-80.10 tractor.

Tests When digging a trench on an experimental machine based on the TTZ-80.10 tractor in the KuyiChirchik region, the irrigation networks were cleaned with earthmoving equipment $[6,7]$.

The air temperature is about $160180 \mathrm{C}$; there is no cloudy weather, precipitation and wind loads.

The excavated soil is a mixture of sand and gravel containing tree roots and category 3 soil, plants, etc.

The test conditions of the multipurpose machine should be classified as severe, especially when clearing the canal, as evidenced by soil control with a $0.3 \mathrm{~m} 3$ excavator bucket. With an "accumulated" bucket, the soil weighed $500-550 \mathrm{~kg}$ and had a density of $1.66-1.83 \mathrm{~g} / \mathrm{cm}^{3}$, and the density of medium compacted soil, typical for Uzbekistan, did not exceed $1.4-1.5 \mathrm{~g} / \mathrm{cm}^{3}$ [8].

The excavator under test belonged to machines with a cyclic mode of operation; therefore, measurements were mainly carried out in the "stop" mode using EMA-P equipment. In this operating mode, it is necessary to determine the average value of the measured parameter in the interval of one second or one minute according to the formulas for subsequent calculations following GOST 300067-93:

$$
P=\frac{m}{t} K
$$

For one minute:

$$
P=60 \frac{m}{t} K
$$

where: $m$ is digital indication of the meter channel.

\subsection{Field experiment results}

As a result of field tests, data were obtained characterizing the operation of the multipurpose machine KM-1 with earthmoving equipment $[8,11]$.

The data obtained show that between works performed by earthmoving equipment, more time is spent on collecting soil, which takes an average of 14.4 seconds and at least 2.3-2.5 seconds to turn the arrow, lower the bucket from the ground and lower the bucket again, the time has been calculated. The rest of the working cycles consume almost the same time and average from 4.0 to $5.0 \mathrm{~s}$.

Measurement of the pump pressure when collecting soil with a bucket, raising a filled bucket, turning the arrow with a filled bucket, pulling the arrow, lowering the soil from the bucket, returning the arrow and lowering the bucket with it showed that the maximum pump pressure of $12.37 \mathrm{MPa}$ arose when the bucket was lifted with soil. This is because the weight of the boom at the center of gravity, the bow, and the filled bucket have a greater impact on the device's energy consumption.

Although there was little change in load during the operations, the pump pressure changed. The pumping frequency changes in the range 28.25-30.15 $\mathrm{s}^{-1}$.

According to the parameters of the measured parameters during technological operations with earthmoving equipment, the results of the energy assessment of the universal machine KM-1 were obtained.

\subsection{Comparison of experimental results with theoretical values}

The results of theoretical and experimental calculations are summarized in the following tables and the calculation schemes. 


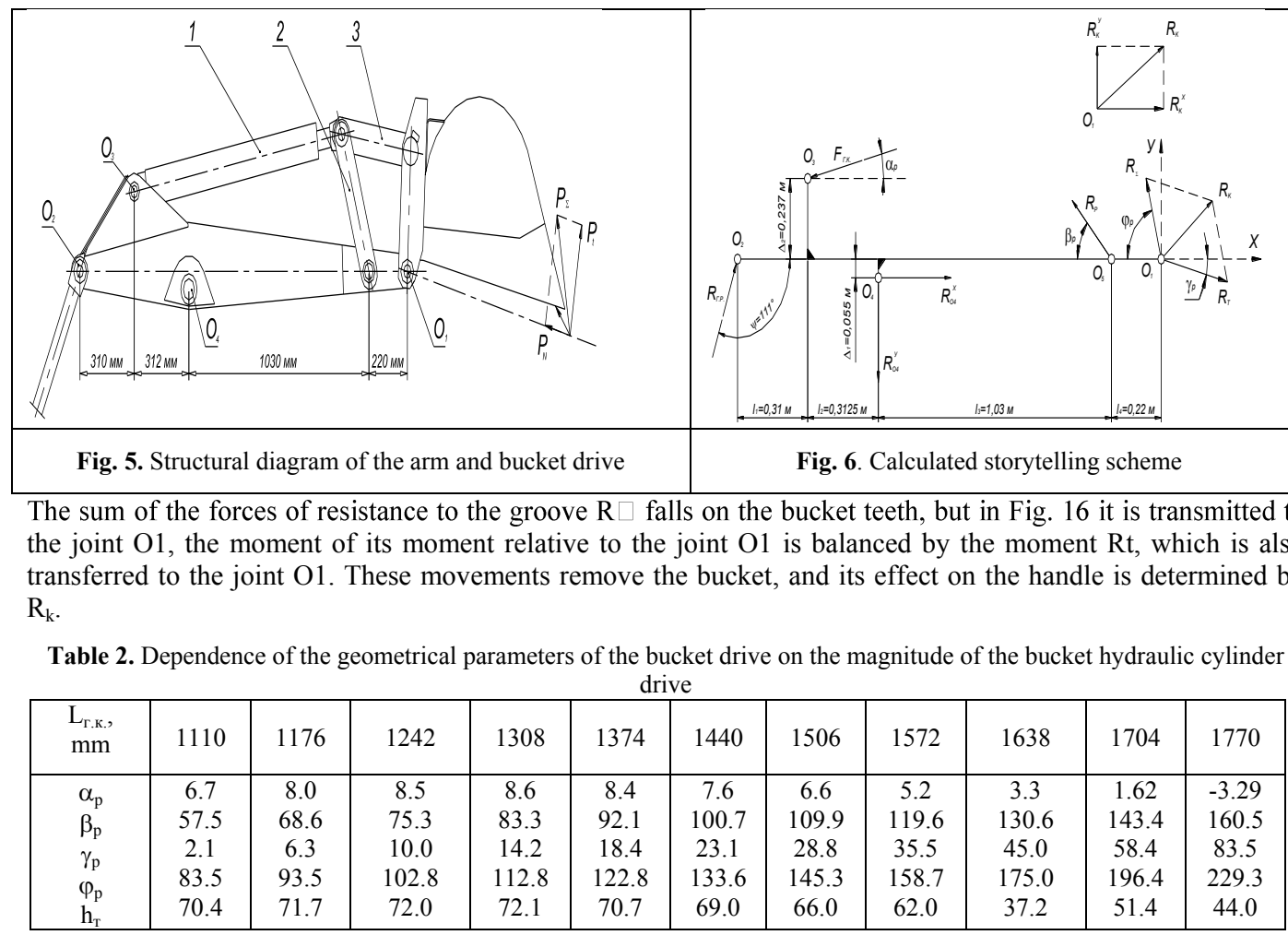

Points are the results of the research. The continuous line is the average line of the survey results considering the excavation process.

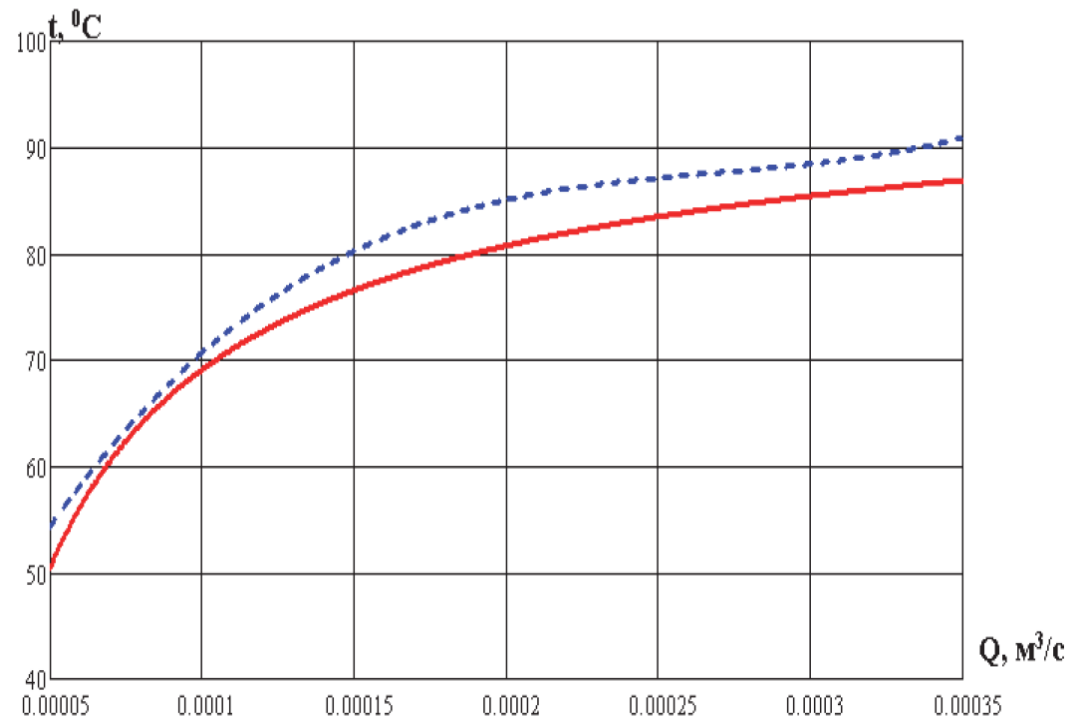

Fig. 7. Comparison of research and theoretical data for drilling $=1000 \mathrm{~S}$ considering the excavation process.

The continuous line is the amount of output of the oil from the theoretical heat exchanger flow rate. The dotted line is the dependence of the flow rate of the oil temperature, leaving the heat exchanger on the research results 


\section{Conclusions}

Tests showed that the pump pressure during soil extraction was $10.76 \mathrm{MPa}$, and the rotational speed was $29.46 \mathrm{~s}$ ${ }^{1}$. At the same time, $25.5 \mathrm{~kW}$ of power was supplied to the installed ground, and the effective engine power was $27.7 \mathrm{~kW}$. The power of the D-245 engine of the TTZ-80.10 tractor was $57.4 \mathrm{~kW}$; during the operations performed, work was performed equal to 0.48 of the engine power factors.

The "maximum" energy losses were observed when lifting the bucket covered with soil, the energy consumption was $28.2 \mathrm{~kW}$, and the engine efficiency was 0.53 .

Almost the same power was spent on stretching the boom with a full bucket and unloading soil from the bucket, which amounted to 24.3 and $22.6 \mathrm{~kW}$, respectively. During these operations, the power factor of the engine is 0.45 and 0.43 , respectively.

The minimum power consumption was $9.9 \mathrm{~kW}$, which was used to return the completed operation to the pit, and the engine utilization rate was 0.20 .

Preliminary tests of earthmoving equipment during felling, harvesting and transportation of soil, the power consumption is $23.42-0.2 \mathrm{~kW}$, which corresponds to an engine power consumption coefficient equal to 0.41 .

\section{References}

1. Action Strategy for the five priority areas of development of the Republic of Uzbekistan for 2017-2021 2017 Approved by the Decree of the President of the Republic of Uzbekistan, (No PF-4947 On the Strategy for further development of the Republic of Uzbekistan).(2017)

2. Augambaev M., Ivanov A.Z., Terekhok Yu.I. Basics of planning a research experiment. (Tashkent Ukituvchi) p 336 . (2004)

3. Afanasyev N.Yu. Computational and experimental methods of scientific experiment: textbooks (MoscowKNORUS), p 336. (2017)

4. Barsky I.B., Anilovich V.Ya., Kutkov G.M. Tractor dynamics. (Moscow "Mechanical engineering") p 520 (1973).

5. Vedenyapin G.V. General method of experimental research and processing of experimental data. (Moscow Kolos) p 199. (1973).

6. Protocol No. 5-2007 state periodic tests of the tractor TTZ 80.10 head. No. 013801. (Gulbahor: UzGCITT) p 87. (2007)

7. Protocol No. 6-2007 state periodic tests of the tractor TTZ 80.11 head. No. 013864. 2007 (Gulbahor: UzGCITT) p 86. (2007)

8. Rustamov K.J. Experimental Work of the Hydraulic Equipment of the Multi-Purpose Machine Mm-1. International Journal of Recent Technology and Engineering 8, (4), November 2019. - pp. 12032- 12036 (2019).

9. Gauges of excess pressure deadweight. (General technical requirements GOST 8291-83).

10. Single-bucket excavator with a bucket $0.25 \mathrm{~m} 3$ on the tractor "Belarus" YUMZ-6AL / 6AM hydraulic EO2621A. (Technical description and instruction manual TO. Saransk excavator plant. Saransk) p 94. (1978).

11. Rustamov K.Zh., Pirnaev Sh.A., Ulmasova L.A. Results of field tests of a multipurpose vehicle. (Modern technologies in the automobile and road complex, the program of the Republican scientific-practical conference), p 3. (2009).

12. Rustamov, K. J. Technical solutions and experiment to create a multipurpose machine. International Journal of Scientific and Technology Research, 9 (3), 2007-2013.Retrieved from www.scopus.com. (2020).

13. Askarhodjaev, T., Rustamov, K., Aymatova, F., and Husenova, G. Justification of the hydraulic system parameters of the excavation body of a multipurpose road construction vehicle based on the TTZ tractor. Journal of Critical Reviews, 7 (5), pp 229-234. doi:10.31838/jcr.07.05.40. (2020)

14. Isyanov, R., Rustamov, K., Rustamova, N., and Sharifhodjaeva, H. Formation of ICT competence of future teachers in the classes of general physics. Journal of Critical Reviews, 7(5), pp 235-239. doi:10.31838/jcr.07.05.41. (2020).

15. Dadhich, S., Bodin, U., and Andersson, U. Key challenges in automation of earthmoving machines. Automation in Construction, 68, pp 212-222. doi:10.1016/j.autcon.2016.05.009. (2016).

16. Feng, H., Yin, C. -., Weng, W. -., Ma, W., Zhou, J. -., Jia, W. -., and Zhang, Z. -. Robotic excavator trajectory control using an improved GA based PID controller. Mechanical Systems and Signal Processing, 105, pp 153-168. (2018). doi: 10.1016/j.ymssp.2017.12.014.

17. Nezhadali, V., Frank, B., and Eriksson, L. Wheel loader operation-optimal control compared to real drive experience. Control Engineering Practice, 48, pp 1-9. doi:10.1016/j.conengprac.2015.12.015. (2016).

18. Jud, D., Hottiger, G., Leemann, P., and Hutter, M. Planning and control for autonomous excavation. IEEE Robotics and Automation Letters, 2 (4), pp 2151-2158. doi:10.1109/LRA.2017.2721551. (2017). 
19. Wang, Y., Shen, T., Tan, C., Fu, J., and Guo, S. Research status, critical technologies, and development trends of hydraulic pressure pulsation attenuator. Chinese Journal of Mechanical Engineering (English Edition), 34 (1) doi:10.1186/s10033-021-00532-z. (2021).

20. Wang, B., Zhou, F., Zhou, H., Ge, H., and Li, L. Characteristics of the fracture geometry and the injection pressure response during near-wellbore diverting fracturing. Energy Reports, 7, pp 491-501. doi:10.1016/j.egyr.2020.12.039. (2021). 